in February 1953, assumed that the theory required that "nebulæ are continually receding into inaccessibility by surpassing the speed of light" 1 . Still earlier, in May 1952, the Astronomer Royal, in describing the theory at a Friday Evening Discourse at the Koyal Institution", said that "at a distance of about two thousand million light years the rate of expansion becomes equal to the velocity of light. At that distance we have, therefore, an observational horizon, beyond which we can never see. . . . As the creation of matter is at the rate needed to keep the mean density of matter in the Universe constant, the rate at which galaxies are being born just balances the rate at which they are lost to view". Neither of these statements, which Mr. Gold's letter now contradicts, seems ever to have been corrected.

If, as Mr. Gold now says, "A change to more sensitive methods of observation would delay the disappearance of any one galaxy", what is the meaning of Hoyle's statement that "the oldest condensation within the observable universe ... has an age of about $1.5 \times 10^{10}$ years"? What particular method of observation corresponds to this figure ?

In view of these and other questions that will doubtless arise when the whole matter is reviewed in the light of Mr. Gold's new explanation, the need seems imperative for a clear and authoritative statement either reconciling the earlier and later assertions or else stating which is to be accepted as the true postulate of the theory.

The Writer of the Article

1 Mon. Not. Roy. Astro. Soc., 113, 404 (1953).

${ }_{2}^{2}$ Proc. Roy. Inst., 35, 336 (1953).

\section{Influence of Liquid Films on Fibre Friction}

Bowden and Tabor ${ }^{1}$ have shown that the adhesive force between a smooth sphere and a smooth plane due to a surface film of liquid is independent of the amount of liquid held between the surfaces. This is not true for the case of a cylinder in contact with a plane, when the adhesive force per unit length of cylinder is

$$
A=4 \vee \sqrt{R / r}
$$

where $R$ is the cylinder radius, $r$ the radius of curva. ture of the liquid meniscus and $v$ the surface tension. According to (1), $A$ will increase as $r$ is reduced by decreasing the amount of liquid present. The adhesion will not become infinite, but its maximum value will be determined by the tensile strength of the liquid or by the surface roughness.

The effect is easily demonstrated for a relatively thick glass fibre adhering to a glass plate by adding water and measuring the adhesion as the water evaporates. With a fibre $0.1 \mathrm{~cm}$. in diameter, values of $A$ up to $2 \mathrm{gm} . / \mathrm{cm}$. are readily obtained. The adhesion cannot, however, be measured directly in this manner for fine textile fibres; but it can be measured indirectly in so far as it modifies the apparent frictional coefficient when rubbing takes place on a smooth surface in the presence of a liquid film.

Fibre frictional coefficients are generally measured by the 'bollard' method for which the well-known relation,

$$
T_{1} / T_{2}=\exp \mu_{\boldsymbol{A}} \theta
$$

is employed, where $T_{1}, T_{2}$ are the fibre tensions, $\theta$ is the angle of contact between fibre and cylinder and $\mu_{A}$ the coefficient of friction. In the presence of an adhesion force $A$, relation (2) will take the form,

$$
\frac{T_{1}+A p}{T_{2}+A_{p}}=\exp \mu \theta
$$

when $\rho$ is the cylinder radius and $\mu$ the true coefficient of friction. Then by measuring values of $T_{2}$ for two different values of $T_{1}$, it is possible to determine both $A$ and $\mu$. Some results obtained using a nylon fibre and a polished glass cylinder are shown in Table 1 . No attempt was made to fix the thickness of the oil film, which was simply reduced in thickness by wiping with a clean cloth. The oil used had a viscosity of about 1 c.g.s. unit and a surface tension of about $30 \mathrm{dynes} / \mathrm{cm}$.; the cylinder diameter was $5.9 \mathrm{~cm}$.

Table 1

\begin{tabular}{|c|c|c|c|}
\hline Oil film & $\mu$ & $A$ & $\mu_{\Lambda}$, from (2) \\
\hline As originally applied & 0.4 & $0.10 \mathrm{gm} . / \mathrm{cm}$. & 0.62 \\
Cylinder wiped once & 0.4 & $0.17 \mathrm{gm} . / \mathrm{cm}$. & 0.79 \\
Cylinder wiped again & 0.4 & $0.26 \mathrm{gm} . / \mathrm{cm}$. & 1.10 \\
\hline
\end{tabular}

In the case of the thinnest oil film, the experiment was repeated using a wool fibre, and the values $\mu=0.4$ and $A=0.03 \mathrm{gm} . / \mathrm{cm}$. were obtained.

It is plain that the surface roughness of the wool fibre resulting from the surface scales has eliminated adhesion effects to a large extent.

\section{G. KnNG}

Wool Industries Research Association, Torridon, Headingley, Leeds 6. Nov. 4.

1 Bowden, F. P., and Tabor, D., "The Friction and Lubrication of Solids", 300 (Clarendon Press, 1950)

\section{Non-linear Microphonic Response of Labyrinth Organs to Stimuli consisting of Two Pure Tones}

IN a study of the microphonic effect of the lateral line of fishes ${ }^{1}$, we found a remarkable response to a stimulus consisting of two pure sine waves acting at the same time on the sense organ. These effects are demonstrated by Fig. 1, curves $A-C$.

The most interesting aspects of these curves are : (1) The frequency of the response $(A$ and $B)$ is twice the frequency of the stimulus. (2) In the combined effect $(C)$, the response to the high frequency is large at the negative peaks and small at the positive perks; the latter correspond (approximately) to the equilibrium position of the organ. (3) The maximum response to the high frequency in $C$ is much larger than if this frequency is presented alone $(B)$, with the same amplitude. The quotient of the two responses will be called the 'amplification'. This amplification depends on the amplitudes of $a$ and $b$.

We are still convinced that the explanation of the double frequency given in our earlier paper ${ }^{1}$ is correct. It is not found in the microphonic effect of the cristæ of the semicircular consls and of the 